Arteterapia. Papeles de arteterapia y educación para inclusión social ISSN-e 1988-8309

http://dx.doi.org/10.5209/ARTE.59899

\title{
Proceso experimental a través de los lenguajes del arte en el abordaje terapéutico de la mujer con trauma
}

\author{
Rosaura Navajas Seco ${ }^{1}$
}

Recibido: 16 de abril de 2018/ Aceptado: 22 de mayo de 2018

Resumen. La implicación social frente a la situación que viven las mujeres que han sufrido maltrato, no es suficiente para dar respuesta a las necesidades requeridas dentro de lo que entendemos por una verdadera inclusión en nuestra sociedad. Ayudarlas en el restablecimiento de sus vidas tanto psíquica como emocionalmente requiere de programas de intervención que incluyan políticas integradoras desde todos los campos. Es importante que no se rompan eslabones en la cadena de ayuda en un sistema de por sí complicado desde que se empieza a hacer una denuncia hasta que la mujer siente que está incluida socialmente como una ciudadana más.

Los programas aplicados a la mejora del estado psico-físico y emocional de la mujer maltratada en gran medida ayudan notablemente a normalizar su situación de vida y relación social. La aplicación de un programa con la intervención de diferentes lenguajes expresivos como son la música, la pintura y la expresión corporal son cruciales en el empoderamiento de estas personas. Nuestra línea de actuación lejos de trabajar tan solo la parte cognitiva en el tratamiento de la mujer, propone una aplicación de programas que acoge en su esencia, la parte cognitiva, afectiva y vivencial mediante actividades cuyo foco nuclear se basa en los lenguajes del arte. Analizaremos un cuerpo de mujer que es consciente, que siente y que actúa para reafirmar su identidad y su relación con el mundo

Palabras clave: trauma y arte; cuerpo expresivo y trauma; programas trauma.

\section{[en] Experimental processes through artistic communication in the theurapeutic approach of women affected by trauma}

\begin{abstract}
The social responses to the situation of those women who have suffered from abuse are not enough to meet their demands, as far as what we understand as a real inclusion in our society is concerned. Helping them recover their own lives (both psychologically and emotionally) requires intervention programmes which shall include inclusive policies in all fields. It is important for the links of the aid chain to remain unbroken in an already complicated system that starts when a woman reports the incident to the police and finishes when she eventually feels socially included as a citizen.

The programmes that aim to improve the emotional and psychophysical status of abused women do remarkably help them to normalise their daily situation and their social relation. The implementation of a programme that includes different expressive types of communication* such as music, painting and body language, is crucial in the empowerment of these people. Our line of action, far from just working on the cognitive area in the treatment of women, proposes an implementation of programmes that include the cognitive, affective and experiential areas at their core, and it does so through different activities that have their focal point on different artistic communication systems. We shall analyse the body of a woman who is conscious, who feels and takes action to assert her own identity and her relationship with the world.

Keywords: trauma and art; expressive body and trauma; trauma programmes.

1 Universidad Complutense de Madrid. Facultad de Educación. Departamento de Didáctica de las Lenguas, Artes y Educación Física

E-mail: rnavajas@edu.ucm.es
\end{abstract}


Sumario. 1. Proceso experimental terapéutico en la mujer que sufre maltrato. Lenguajes del arte (Música, Pintura y Expresión Corporal). 1.1. Emoción y líneas de pensamiento. 1.2. Lenguajes artísticos y el sentido terapéutico. 1.3. Programas de intervención. 2. Matriz guía para la intervención de procesos terapéuticos en mujeres maltratadas. 3. Conclusiones. 4. Referencias bibliográficas.

Cómo citar: Navajas Seco, R. (2018). Proceso experimental a través de los lenguajes del arte en el abordaje terapéutico de la mujer con trauma, en Arteterapia. Papeles de arteterapia y educación para inclusión social 13, 2018, 33-52.

\section{Proceso experimental terapéutico en la mujer que sufre maltrato: lenguajes del arte (musica, pintura y expresión corporal)}

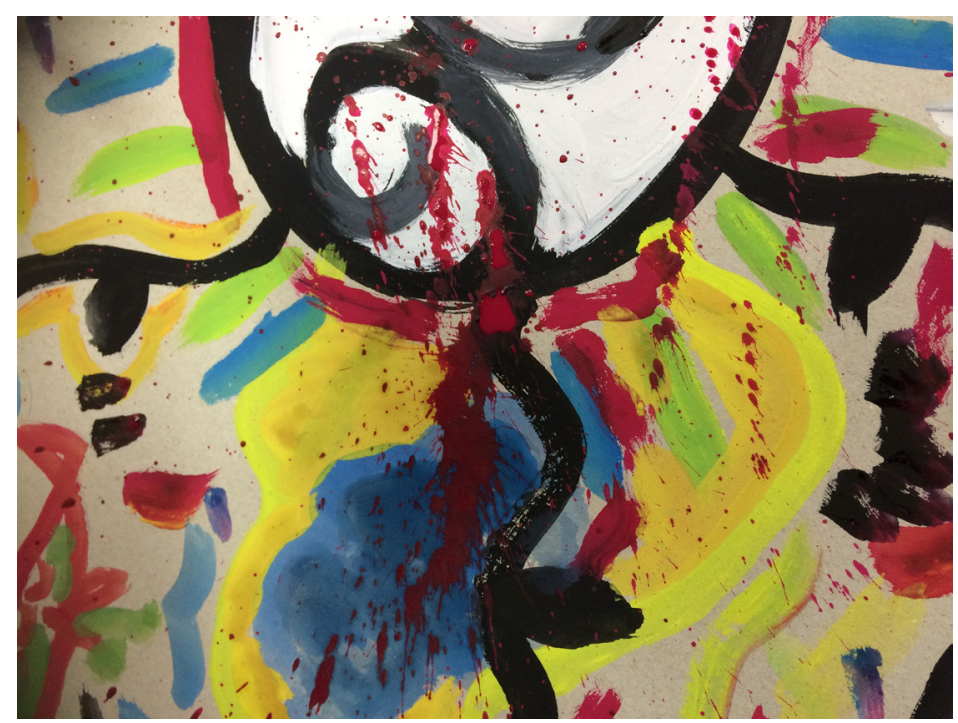

Fig.1

\subsection{Emoción y líneas de pensamiento}

Las teorías emocionales ya desde el siglo XIX, constatan que los sentimientos, las sensaciones, las formas de expresar los procesos internos de pensamientos, están íntimamente relacionados con manifestaciones corporales. La teoría del sentir nos acerca a analizar aspectos cualitativos de la persona desde un modelo de percepción que se centra en el estado corporal y sus múltiples manifestaciones ante un suceso. Cualquier sensación de la índole que sea se transforma en sensaciones y estados que se expresan de forma fisiológica. El corazón, el estómago, la respiración, y otros órganos y procesos fisiológicos están íntimamente relacionados con lo que le está pasando al individuo dentro de un estado emocional determinado. Este pensamiento está relacionado con la teoría evolutiva y fisiológica de (James, 1991). Pero la forma en la que las emociones nos afectan, no están contempladas en este tipo de pensamiento, que va más en la línea de (Damasio, 2001), donde nuestras emociones, no solo condicionan nuestras percepciones, sino nuestra ex- 
periencia de la realidad. Esta experiencia de la realidad está basada en creencias y las posibles prioridades que tengamos en la vida.

Tanto el modelo de percepción como el modelo de juicio, tienen sentido a través de la vivencia y experiencia de la persona. Por lo tanto, si existe experiencia y ésta se hace consciente, lógicamente tiene que ir acompañado de algún tipo de proceso mental. Es decir la sensación de odio, asco, ira, rencor y otras muchas sensaciones se manifiestan corporalmente proyectadas con procesos fisiológicos muy diversos, como palpitaciones, sudores, presión en el estómago, etc. Por lo tanto, de alguna forma la mente ha conectado con situaciones que le han llevado a ser consciente de su estado y de las manifestaciones corporales que se están dando en el cuerpo de la persona. Se podría comprobar que este fenómeno está relacionado con la percepción consciente de los estados de ánimo según Prinz (2004).

Cada sensación, traducida en un estado fisiológico corporal tiene diferenciación en sus manifestaciones, por lo tanto la persona, haciendo un análisis consciente y vivencial, puede observar lo qué le está ocurriendo a su cuerpo dependiendo del tipo de afección sentida, sobre todo a nivel visceral. "son las cualidades sentidas las que cumplen con la función de diferenciar una emoción de otra" Vendrell, (2008 pag.220). Otras líneas apuntan a relacionar las emociones con los juicios, se habla de una razón de dependencia que estará sujeta a diferentes situaciones que pueden ser cambiantes, es decir que al cambiar un juicio sobre cierta emoción también cambia ésta. Taylor (1985). Por el contrario. estudiosos de la emoción consideran que el juicio no tiene por qué ir unido a la emoción y que se pueden dar absolutamente separados. Añaden que se puede emitir un juicio sin que este llegue a producir ningún tipo de reacción, por lo tanto, no solo olvidan la emoción sino las reacciones corporales de las teorías del sentir. (Goldie 2002).

\subsection{Lenguajes artísticos y el sentido terapéutico}

El trabajo con diferentes lenguajes artísticos proporciona al ser humano una forma de conocer el mundo desde distintas perspectivas, ya que son expresiones que nacen y surgen de las sensaciones que les proporciona tanto la música, pintura, como el movimiento. En situaciones donde se aplican programas que incluyen diferentes formas de afrontar el trauma con procesos de desarrollo artísticos, se incide en que el clima sea foco de atención en el abordaje de cualquier actividad. La creación de un espacio de seguridad, es fundamental para que la persona libere pensamientos internos y se generen momentos donde puedan sentirse en la intimidad, y empatizar con las personas de su entorno (Lynn, 2001). La desconfianza forma parte de la situación emocional de la persona, generando situaciones de evitación continuamente en la interacción incluso con sus más allegados. La tendencia al aislamiento se hace evidente dentro del sufrimiento que entraña la consideración de hacer una generalización desde que se sufre el trauma, incluyendo sin ser consciente hábitos antisociales o conductas de alejamiento. El contacto, la cercanía y la expresión de cualquier situación donde tenga que ponerse en contacto con otros supone emociones encontradas. (Cordoba, 2013)

Cuando la intervención corporal en los procesos terapéuticos se hace tangible, y proporcionamos al cuerpo movimiento y creación, además de libertad de movimiento, con espacios para sentir, la expresión interior de nuestros sentimientos se hace externa, es por tanto cuando se hace consciente la importancia de vivir el movimiento y el cuerpo a través de múltiples recursos como la música y la pintura generando espacios de encuentro en la persona, (Penhofer, 2005). 
Los procesos de mejora en la adquisición de competencias olvidadas en la mujer, más la capacidad de poder volverse a relacionar con las personas de forma saludable y recobrar cierto grado de autoestima, merece ser tratado a largo plazo para que se afiance todas las vivencias del proceso, García (2012). Tanto la pintura, como la música y danza, necesitan tiempo para que se vayan consolidando como herramientas de trabajo. Callejón y Granados (2003), nos hablan sobre la importancia de trabajar desde la infancia todas las artes, e incluso no dudan en expresar que si todo el mundo de alguna forma necesita ayuda, por qué no se puede trabajar de forma terapéutica en la escuela. Hablan de la escuela Waldorf como ejemplo, cuya filosofía está relacionada con el trabajo sobre el arte, la razón y el juicio como una forma de despertar en la vida.

La música, al igual que la danza y la pintura, consiguen canalizar las emociones y poder liberarlas hacia el exterior. Son lenguajes universales que armonizan de una forma integral todos los sistemas que el cuerpo tiene, junto con lo que nos pueden transmitir frente a nuestros estados de ánimo. La parte motriz de la persona es la que exteriorizará todo lo que de alguna forma puede aportar la música y la danza cuando ponemos todo nuestro interés en dejarnos llevar por las sensaciones que nos producen estos dos lenguajes Las artes pueden ayudar a conectar con los lazos familiares, además que en ellas existen componentes culturales que hacen que seamos capaces de ponernos en conexión con la sociedad. (Otero y Fernández, 2017),

\section{3.- Programas de intervención}

Otero y Fernández (2017), a través de experiencias artísticas contadas por los participantes, se generan unas narrativas que dan lugar a revivir, crear y generar un entramado de historias que dan sentido a identidades. Se basan en la interrelación de arte, el afecto y la autoexpresión aplicado en entornos sociales y culturales. Otros programas aplican un trabajo sobre la identidad y autorretrato a partir de experiencias artísticas. Es ahí, donde se observa que surge un sentimiento de autoconciencia, además de que la experiencia conecta de lleno con diferentes emociones que les llevan a promover cambios considerables en la persona. Bascones (2013).

(Ojeda y Serrano, 2008), trabajan con un grupo de mujeres gaditanas que sufren maltrato y uno de sus propósitos está relacionado, con la búsqueda de espacios donde sean capaces de asumir el enfrentarse a la superación de miedos. Se basan en la importancia de la reconstrucción de estructuras defensivas donde se puede observar la baja autoestima y la propia pérdida de identidad. A través de un trabajo de arteterapia consiguen tener mayor grado de madurez sobre sus acciones y sus vivencias y emociones. Parece haberse encontrado diferencias positivas, entre el pre y post tratamiento en casi todos los ítems, tanto en la depresión, como ansiedad, autoestima e inadaptación a la vida cotidiana.

Otro tipo de intervenciones relacionado con el Trastorno de Estrés Postraumático, es el de (Alonso, 2007), en donde se valida empíricamente un programa de tratamiento para la mujer víctima de violencia doméstica. En este proceso se observan mejoras en la autoestima, y en el seguimiento de pautas para casa, donde la mujer sigue trabajando para fortalecer acciones que estaban olvidadas. El programa incluye actividades gratificantes además de haber sido muy exitoso por el nivel de participación de las personas en él.

Nieto, Concepción y Casares (2016), aplican un programa psicoterapéutico, donde atienden a personas que han sufrido experiencias traumáticas complejas. El valor de esta psicoterapia es que atiende no solo a la sintomatología psicobiológica, sino que llevan un seguimiento en cuanto a la implicación social, sobre apoyos de cara a 
una inserción social. Su objetivo es que la persona sea consciente de aquellos patrones que se repiten corporalmente y que afectan a la proyección de la imagen. Por lo tanto su misión es modificarlos cuando estos puedan afectar a su actividad diaria y así poder incorporarse a la normalidad de sus vidas cuanto antes.

Estos y muchos programas de intervención están llevados con gran éxito desde proyectos, asociaciones, y entidades que quieren dar respuesta a esta situación. Pero como se dijo en la introducción, no es suficiente el trabajo aislado que se pueda hacer por instituciones, sino que hay que afrontar de una forma integral y global el problema para una verdadera inserción social.

\section{Matriz guía para la intervención de procesos terapéuticos en mujeres maltratadas}

En esta tabla se incluye por orden las distintas partes del proceso. Comenzamos con una primera parte cuyo trabajo va más dirigido al desarrollo de aspectos cognitivos del lenguaje, donde la persona tiene que ser capaz de tener una buena comunicación con sus interlocutores para poder entender con más claridad todos los estados internos de pensamiento que Le están sucediendo. Con una buena comunicación hay mayor viabilidad en la interpretación por otros de su situación. Así se desarrollan actividades de tipo, semántico, sintáctico y léxico, para trabajar las estructuras base de una buena comunicación. Quiere decir que el primer lenguaje que afrontamos es el propio comunicativo usado por la persona de forma habitual. En la segunda parte pasamos a trabajar otros lenguajes más artísticos, donde la mujer maltratada tendrá la oportunidad de liberar sus pensamientos, sensaciones y anhelos a través de la pintura y la expresión corporal.

De esta manera se desarrolla un trabajo donde intervienen distintos lenguajes que refuerzan y mejoran el estado de la mujer de forma integral.

Tabla1. Elaboración Propia (Navajas, 2018)

\begin{tabular}{|c|c|c|}
\hline \multicolumn{3}{|c|}{$\begin{array}{c}\text { PROCESO EXPERIMENTAL TERAPÉUTICO } \\
\text { EN LA MUJER QUE SUFRE MALTRATO } \\
\text { LENGUAJES DEL ARTE (MUSICA, PINTURA } \\
\text { Y EXPRESIÓN CORPORAL) }\end{array}$} \\
\hline PRIMERA PARTE DEL PROCESO & \multicolumn{2}{|c|}{ SEGUNDA PARTE DEL PROCESO } \\
\hline $\begin{array}{l}\text { DIMENSIÓN } \\
\text { COMUNICATIVA }\end{array}$ & \multicolumn{2}{|c|}{$\begin{array}{l}\text { DIMENSIÓN } \\
\text { EXPRESIVA }\end{array}$} \\
\hline $\begin{array}{l}\text { COMPONENTE COGNITIVO } \\
\text { Pienso: hablo }\end{array}$ & \multicolumn{2}{|c|}{$\begin{array}{l}\text { COMPONENTE CONATIVO Y EXPRESIVO } \\
\text { Siento y actúo: pinto y bailo }\end{array}$} \\
\hline HABLA & PINTURA & $\begin{array}{l}\text { EXPRESIÓN } \\
\text { CORPORAL }\end{array}$ \\
\hline $\begin{array}{l}\text { ATENCIÓN PLENA } \\
\text { EN LA COMUNICACIÓN }\end{array}$ & \multicolumn{2}{|c|}{$\begin{array}{l}\text { MÚSICA } \\
\text { TANSVERSAL }\end{array}$} \\
\hline \multicolumn{3}{|c|}{$\begin{array}{c}\text { EL DEBATE } \\
\text { TRANSVERSAL } \\
\text { EN TODO EL PROCESO TERAPÉUTICO }\end{array}$} \\
\hline
\end{tabular}




\section{PRIMERA PARTE DEL PROCESO}

\subsection{Dimensión comunicativa: componente cognitivo}

(Pienso: Hablo)

En la primera parte del proceso y en los primeros contactos con las mujeres maltratadas se establece un dialogo que permita conectar con ellas y desarrollar un clima agradable y de confianza. Esta fase comunicativa es crucial, porque marcará incluso el abandono de personas si no se han sentido cómodas en el lugar. Por tanto es el momento de establecer relaciones afectivas a través de una interacción calmada y tranquila entre la persona que lleva el programa y las mujeres que han sufrido maltrato.

En relación con el comienzo de la fase comunicativa y la interacción verbal entre todo el equipo, hay situaciones que se suelen repetir de forma habitual como un cliché:

- Dificultad para llevar una comunicación fluida y continuada, con parones en la comunicación por considerar que no saben hablar.

- Desorden de estructura en las frases que aclare de forma nítida cual es el foco temático de la conversación.

- Bloqueo emocional, por tanto suele seguir un bloqueo en la emisión de las palabras

- La timidez de no expresar claramente lo que sienten, por miedos a no ser escuchadas o por considerar que su palabra va a ser contradicha o no tomada en cuenta.

- Distorsión y desorden en el pensamiento con lo que a veces es difícil transcribir la idea fundamental o incluso no son capaces ni de decirlas.

- Muchas onomatopeyas cuando hablan, sustituyendo a las palabras.

- Repetición de la misma palabra y silencios, que van unidos con una imagen gestual de impotencia.

Está claro que en el lenguaje se reflejan disfunciones cerebrales y en este caso vemos como personas que han estado y están sometidas a maltrato continuado presentan estos patrones y actitudes reflejados en los puntos anteriores. Por ello no solo es necesario que se establezca un clima tranquilo y de confianza, sino que también la persona que guía el coloquio o el debate en el grupo, tiene que presentar ciertas actitudes y comportamientos tales como:

- Escucha plena

- Gestos de refuerzo positivo

- Palabras de apoyo

- Evitar que la persona no sienta espacios vacíos de comunicación

- Incorporar en diferentes momentos en el debate a personas del equipo que no hablan

- Si afloran emociones dejar que se expresen con libertad

- Mantener un tono agradable sin altibajos en la voz 


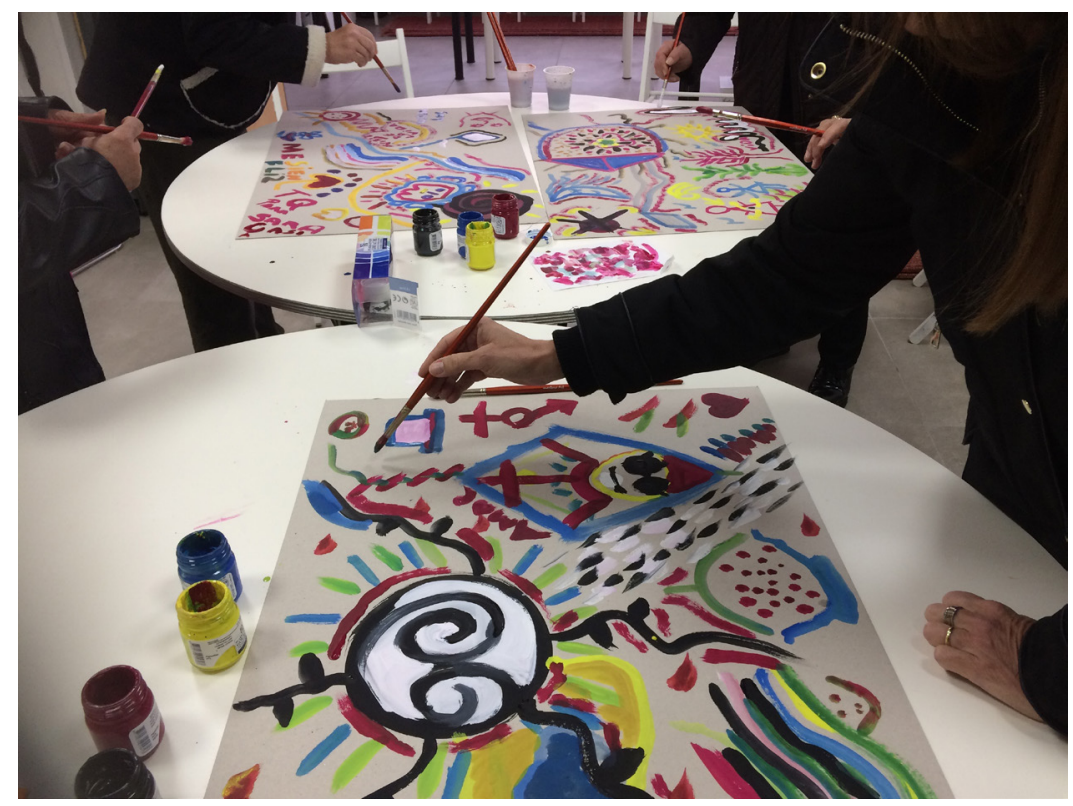

Fig.2

En este apartado se han desarrollado actividades para mejorar las dificultades comunicativas que tienen estas personas a la hora de expresar sus sentimientos y sensaciones. Este tipo de talleres hacen uso de ciertas herramientas y estrategias para que las mujeres sean capaces de comunicar de forma libre todos aquellos aspectos del pensamiento interno.

\section{Explicación de la actividad nuclear desarrollada en el programa:}

Objetivos de mejora comunicativa en la mujer:

- Enseñarles a construir frases con orden, poniendo el foco de atención sobre lo que quieran expresar.

- Ser capaces de comunicar con claridad los sentimientos y sensaciones

- Tener una comunicación fluida, sin frenos ni miedos

- Tener confianza en una misma, para poder hablar con asertividad frente a los demás

- Confiar en el equipo de mujeres y escuchar a las otras respetando los tiempos

- Ser capaces de interpretar otros sentimientos en la comunicación y dar consejos u opiniones a sus compañeras.

\section{Pasos del proceso}

- Se les dice que formulen una frase escrita donde se marquen un objetivo que les acerque a cumplir en el futuro algún sueño. En este apartado tienen que formular bien la frase y expresarla con tranquilidad y fluidez. 
- Todas deben leer frente al equipo su frase, y comentar el por qué de ese sueño. Es una manera de acercarnos a sus historias de vida y a conocer aspectos que le puedan ilusionar a la persona. En esta fase comienzan a abrirse y a sentir que son escuchadas por los demás.

- Una vez que se han escuchado, se les pregunta sobre las posibilidades de cumplir sus sueños.

- Analizan qué cualidades son las que tienen para llegar a ese objetivo. Y también que aspectos de ellas pueden hacer que el objetivo se vaya cumpliendo con dificultad.

Con este tipo de tareas, están haciendo un trabajo reflexivo-mental, donde se pone atención en el orden de exposición, y se van expresando libremente creando espacios de confort y seguridad. Se tiene en cuenta toda la parte expositiva y el nivel de interacción de cada persona. De manera fluida se van incorporando cada vez más aquellas personas que mostraban timidez.

Esta actividad reina se puede repetir en diferentes momentos del programa, pero con distintas temáticas y hemos comprobado que son actividades muy significativas e importantes para ellas.

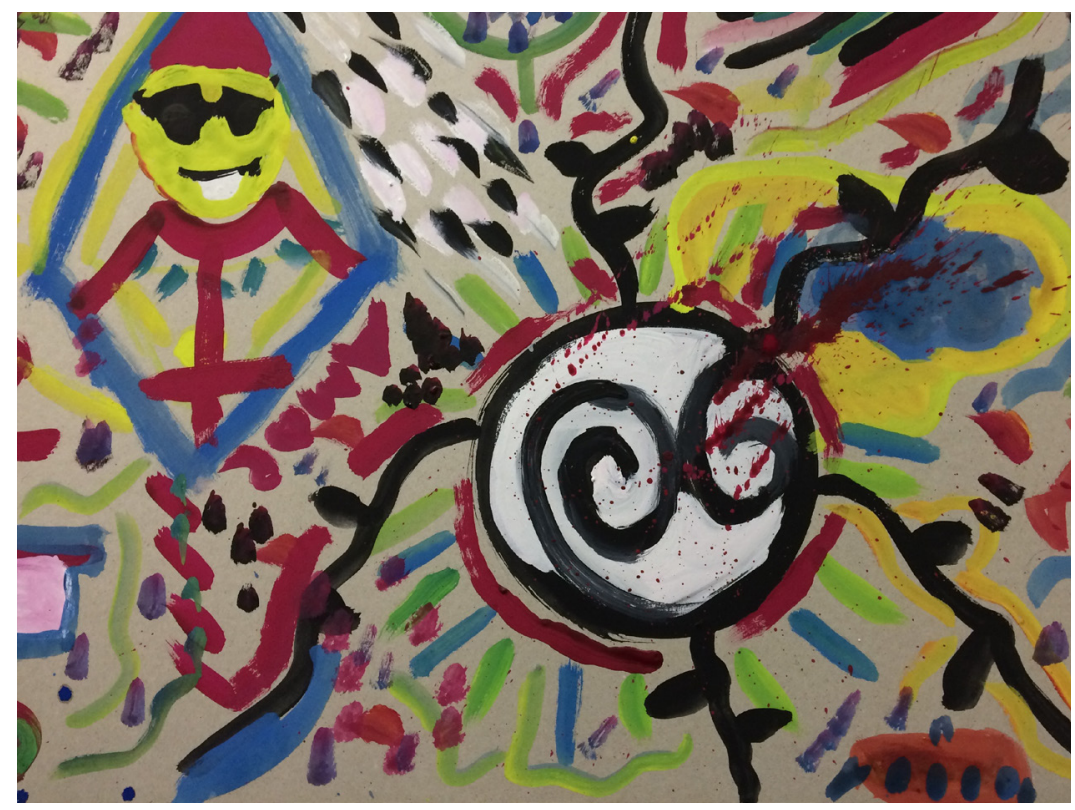

Fig.3

\section{¿En qué mejoran las mujeres con este tipo de actividades?}

- Seguridad en ellas a la hora de pensar y expresarse, sobre todo ante los demás

- Exposición de ideas ordenadas y claras

- Comunicación fluida y cómoda

- Pérdida de vergüenza cuando se enfrentan a un público

- Capacidad asertiva para comunicarse con los demás

- Ampliar su círculo comunicativo en otros espacios y con diferentes personas 


\title{
SEGUNDA PARTE DEL PROCESO
}

\subsection{Dimensión expresiva: componente conativo y expresivo}

\author{
(Siento y Actúo: Pinto y Bailo)
}

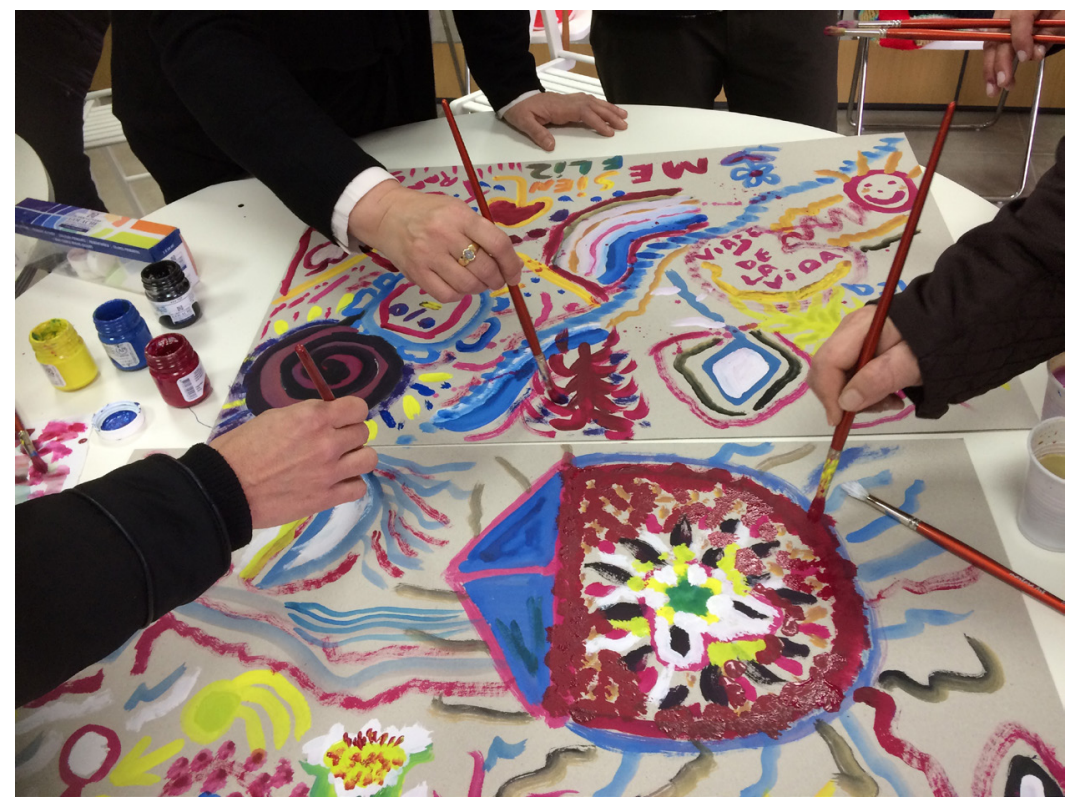

Fig. 4

El programa propone la intervención de varias disciplinas como son la música, pintura y expresión corporal para trabajar sobre estados de ánimo, sentimientos encontrados y emociones que evocan hechos del pasado y del presente. Es una manera de comunicar al mundo y externalizar aquellas sensaciones que necesitan salir y ser liberadas desde diferentes lenguajes expresivos.

\section{EL LENGUAJE PICTÓRICO}

Después de haber trabajado durante varias sesiones aspectos comunicativos fundamentales para descargar sensaciones, estados de ánimo, vivencias y actitudes con la palabra. Pasamos a la parte pictórica del proceso, donde todas las mujeres se prepararán para pintar y ser creadoras de una obra en conjunto con todo el equipo.

Puesta en marcha de la creación.- Esta primera arrancada hacia una forma de expresión poco habitual como es el lenguaje del pincel, es de gran interés porque se presenta sorpresivo y nuevo. Todas parten de cero intentando entender que todo lo que plasman debe ser producto de su sentir, de la emoción y la música que escuchan. Se puede observar cómo los comportamientos son diferentes a la hora de abordar el lienzo. El temperamento, la personalidad y las sensaciones, de cada mujer hacen de 
esta situación un momento mágico de apertura y de entrada a un mundo nuevo. Las diferentes reacciones presentan cuerpos expresivos distintos.

- La mujer que encuentra un espacio cálido y de libertad y dibuja libremente sin prejuicios

- La persona impulsiva que traza su dibujo con firmeza y rapidez

- La persona rezagada que observa a las otras y se espera para empezar su obra de forma distante frente al lienzo.

- Aquella que necesita que le ayuden a colaborar en la obra

- Y la que no quiere pintar porque su aportación cree que no será valida

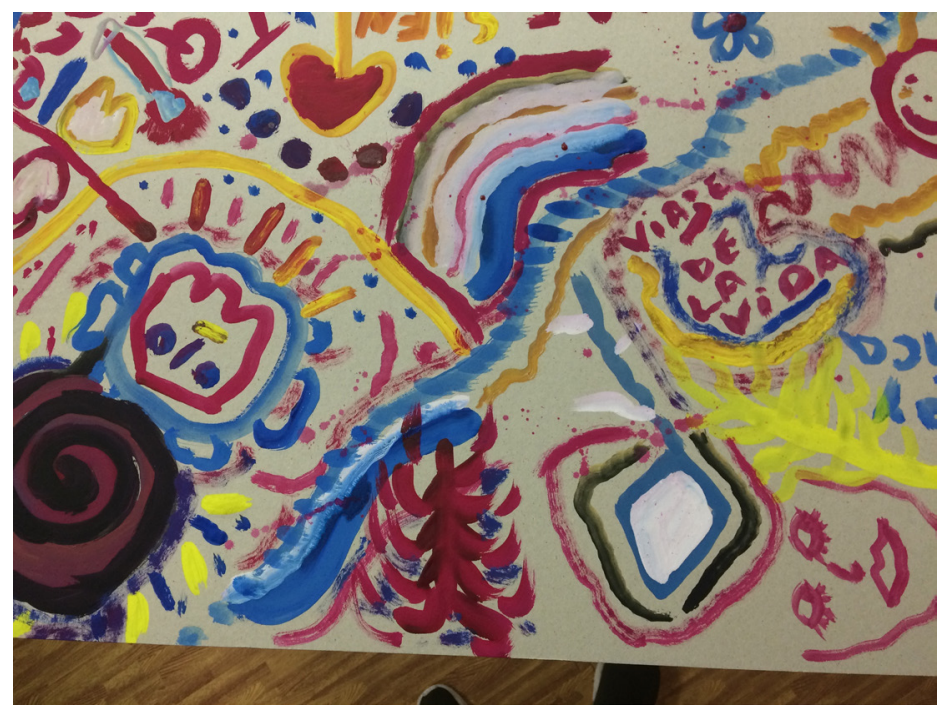

Fig.5

Una vez que se establece un espacio intenso en emociones al ver las creaciones, la guía comienza a hacer preguntas sobre el proceso de creación y las sensaciones emergentes:

Al comenzar.- Cuando habéis empezado a pintar sobre el lienzo

- ¿Qué sensaciones habéis tenido?

- ¿Era difícil arrancar a pintar poniendo los primeros símbolos?

- ¿Os fijabais en vuestras compañeras?

- ¿Había sensaciones que os bloqueaban? ¿Cuáles?

- ¿El no conocer este lenguaje os daba inseguridad?

\section{Paralelismos encontrados al comenzar la obra con situaciones transferibles a la vida real en la mujer que sufre maltrato:}

Si analizamos la situación que se da cuando empiezan a poner sus primeras pinceladas en el lienzo, es como enfrentarse a un parto primerizo. Estos momentos son trasferibles en la vida real cuando tienen que enfrentarse a situaciones por primera vez. desde las sensaciones que sienten al enfrentarse a una situación nueva, como 
bloquearse y no poder actuar de forma tranquila por estar fuera de su zona aparente de confort. La inseguridad y la pereza de moverse en contextos nuevos es una característica muy repetida en la mujer que sufre maltrato. Por ello todo tipo de acciones que existan en el programa para romper el hielo y pasar a entornos no conocidos tienen mucho significado en la mejora de su calidad de vida. Expresar las sensaciones que sienten al bloquearse y observar las actitudes corporales que adoptan en esos momentos de bloqueo son de gran interés y de análisis para ellas.

El Proceso de la creación.- Durante el proceso de creación cada persona va aportando al lienzo sus símbolos, unos entendibles y otros no. Poco a poco se va observando como la tensión va perdiendo sentido y da paso a un espacio liberador haciendo que las mujeres más tímidas participen de forma relajada. Cada vez los símbolos se agolpan e incluso se permite pasar un trazo sobre otro, cambiar los colores de algún dibujo o complementarlo. En este proceso hay un momento de apertura comunicativa en el equipo que es muy importante abordar. La guía entra a dinamizar espacios en donde se van a verbalizar situaciones que están siendo modificadas en sus vidas desde que empezaron con el programa. Entre ellas se animan y valoran el trabajo que están haciendo de cambio en su entorno. Expresan de forma concreta acciones que han cambiado para bien, y los sentimientos que han aflorado por afrontar con éxito esas situaciones. Paralelo al cuadro que están pintando entre el equipo, junto con las conversaciones que se están tratando, aparecen otros lenguajes expresivo corporales que enfatizan en cada mujer el cambio que se está experimentando en ellas. De forma asertiva acompañan la palabra con el cuerpo, y reafirman que se está haciendo una transformación en ellas. Nacen expresiones de alegría y aplausos, abrazos y besos, expresiones positivas y afirmaciones de cambio. Todo un momento de júbilo y de credibilidad porque el cambio puede ser posible.

Durante la creación de la obra, se produce una situación emocional donde la tensión va dejando su espacio a estados de tranquilidad y armonía. Las mujeres escuchan la música y se refleja en su rostro y en su actitud corporal relajación y fluidez.

En el proceso.- Durante la fase de creación de vuestra obra.

- ¿En qué momento os habéis sentido más libres para pintar?

- ¿Os ha sido grato hacer una obra junto con vuestras compañeras?

- ¿Qué tipo de sensaciones y emociones habéis tenido en todo este proceso?

- ¿Habéis colaborado entre vosotras?

- ¿Habéis sentido fluidez cuando pintabais?

\section{Paralelismos encontrados en el desarrollo de la obra con situaciones transferibles a la vida real en la mujer que sufre maltrato:}

Cuando analizamos el proceso de creación vemos cómo se van modulando las intensidades. Con el trabajo de intervención comunicativa, las mujeres también hacen conscientes sus inquietudes y plasman su pasado y presente a golpe de pincelada como respuesta a sensaciones de rabia, ira, impotencia y alguna más que se puede vislumbrar. Hay una fase comunicativa profunda entre ellas, que muestra la comprensión entre los estados emocionales que afloran y la pintura que realizan en colaboración. Todos los sentidos están atentos y la actividad cobra una importancia suprema en el aprendizaje y mejora de los estados psíquicos que podría adaptarse a cualquier acción de la vida real. 
Fin de la creación.- De forma natural el equipo va decidiendo que los lienzos están terminados. Sin decirles nada todas ellas van a lavar sus pinceles y los vasos como fin del producto. La sensación que le invade al guía es bastante simbólica, ya que es una situación edificante que necesita de un ritual. Limpiar los pinceles, vasos y mesas requiere de un momento tranquilo de cierre. Todo dispuesto para admirar las obras terminadas en un espacio ordenado y limpio, donde las participantes observan sus creaciones y experimentan alegría por haber podido participar con sus compañeras en construir una obra bella y llena de sentido.

En el proceso final, existe una especie de catarsis que lleva al equipo a un estado de alegría por haber hecho un trabajo con resultado final artístico bonito. El llegar a ver la obra finalizada es como el descanso del guerrero, que piensa que ya finalizó su batalla con éxito y solo queda descansar. El observar la obra terminada y el final de la construcción de algo que no existía da pie a que nazcan sentimientos de incredulidad, de irrealidad de lo real o de embriaguez. Saltan preguntas como ¿esto lo he podido hacer yo? ¿es posible? ¿he podido colaborar con más personas en la construcción de una obra pictórica?.

Al finalizar.- Es importante cerrar el ciclo y preguntar sobre la obra realizada

- ¿Cuál es el sentimiento o los pensamientos que se os presentan al observar la obra terminada?

- ¿Creíais posible terminar la obra?

- ¿Es interesante haber llegado hasta aquí, viendo un producto final al terminar un proceso?

- ¿Qué sensación tenéis al haber colaborado juntas en la construcción de una obra llena de contenido?

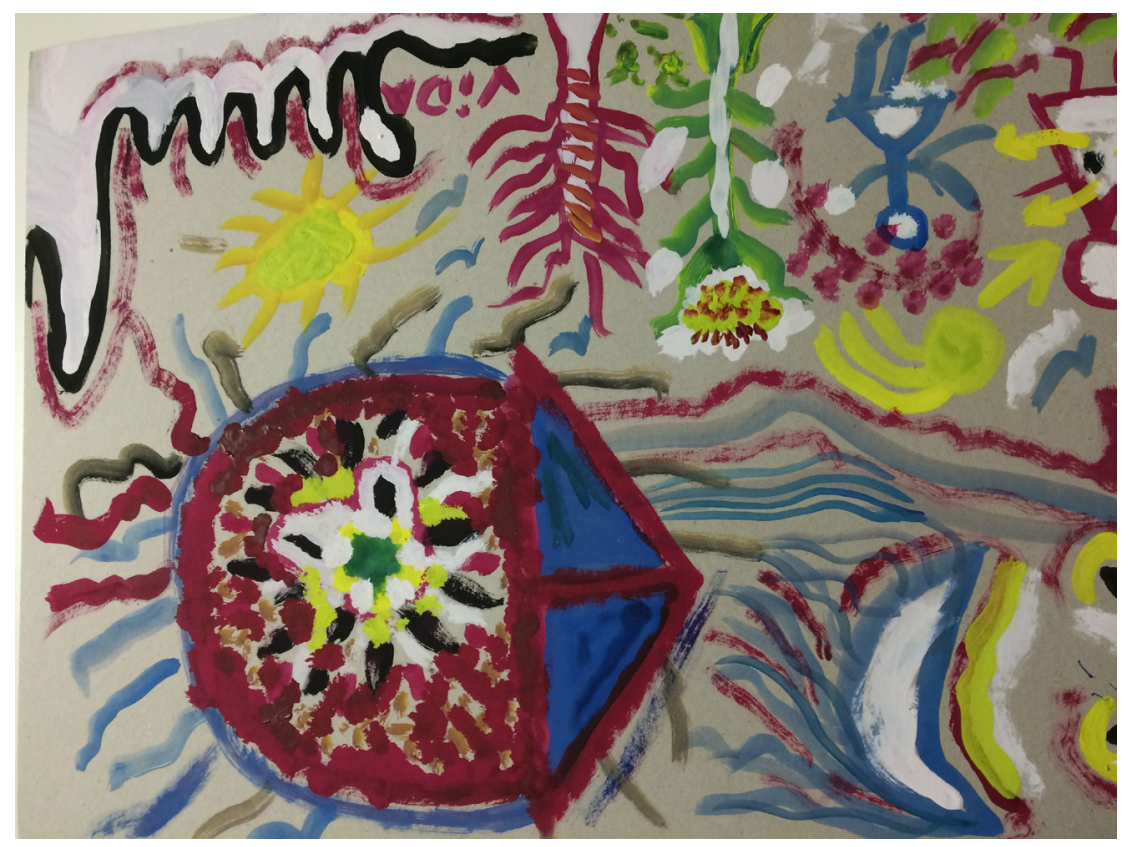

Fig.6 


\section{Paralelismos encontrados cuando la obra está finalizada con situaciones transferibles a la vida real en la mujer que sufre maltrato:}

Una obra finalizada, es la consecuencia de un trabajo hecho. Uno de los sentimientos que se repiten en la mujer que sufre maltrato es en muchos casos la poca ilusión en empezar y finalizar algo. El sentimiento de impotencia por abandonar, evitar, huir de procesos que necesitan evolución y cuidado. Procesos que están cargados de respeto, como es el trabajar en colaboración con otras mujeres cuyas representaciones pictóricas son respuestas de vivencias, y sensaciones pasadas. Hechos que han marcado sus vidas y que ahora llegan a plasmar en un lienzo con valentía y dolor. La obra finalizada responde a un logro, a un progreso, es conseguir un reto, abre puertas de libertad. Se consiguen sentimientos como; yo puedo, soy capaz, lo he conseguido, si he hecho esto puedo seguir logrando objetivos. Todo logro es consecuencia de un esfuerzo, dejar a un lado la procrastinación. La obra finalizada indica éxito, y cuando el éxito se consigue con más personas, es un alimento mayor para el espíritu y se generan estados positivos sobre las ganas de vivir y salir fortalecido.

Análisis del lenguaje pictórico de las obras realizadas y debate con las mujeres: Se hace una puesta en común con todas las mujeres sobre las obras artísticas realizadas. En torno a la mesa y los lienzos pintados se verbaliza aquéllos símbolos, o formas concretas que han sido pintadas por cada una de ellas. Manejan el lenguaje comunicativo a través del cual explican sus dibujos y conectan libremente con hechos, sensaciones y sentimientos ocurridos. Se apoyan entre ellas y disfrutan de la obra, porque hacen patentes aquellas realidades escondidas y que afloran sin ningún tipo de miedos, ni juicios, ni excusas. Todas estas expresiones se dan en una realidad objetiva que intenta valorar el momento en el que están y las circunstancias en la que viven. Desde la parte más técnica del análisis se les explica la importancia de la línea, la luz, los colores, la composición, la situación en donde están dispuestos sus dibujos. Este punto es muy interesante porque se les enseña a analizar el valor emocional de su pintura, además del valor representativo de sus trazos. Descubren un mundo fascinante de interpretaciones al explicarles sobre el trazo y sus características en relación a las personalidades. También se les habla del color y de la elección de estos en conexión con los estados emocionales percibidos.

\section{EL LENGUAJE CORPORAL}

Una vez terminada la parte pictórica, entramos en la fase expresivo corporal, donde las mujeres expresan de forma libre y con su cuerpo movimientos transcritos de las imágenes de los lienzos. Es un espacio donde utilizan todas las partes de su cuerpo, vibrando con la música, realizan trazos con sus movimientos y expresarán los colores con la emoción de sus gestos y las miradas. La actitud frente a la música y la pintura revelan un estado de tranquilidad y calma, pero cuando se incorpora el movimiento, en este caso parecido a la danza expresiva, biodanza $u$ otros movimientos expresivos libres cobra un interés liberador. En un principio cuando se realiza este tipo de actividades existe cierto grado de timidez, pero a medida que va pasando el tiempo el equipo de mujeres va cogiendo confianza y sienten una sensación de confort muy agradable. La fusión de todos los lenguajes artísticos hasta llegar al movimiento tiene gran importancia, ya que se disponen muchos sentidos a la vez como son, la vista, el oído y el tacto. Esta conciliación de sentidos y expresión de liberad 
frente a la cantidad de pensamientos y sentimientos que se agolpan para salir, en un espacio tranquilo y seguro hace que emerja de forma espontánea la emoción a través de lloros, abrazos, gritos, suspiros, movimientos impulsivos y expresiones de dolor y rabia, junto con otros de alegría y felicidad. Son sesiones catárticas y llenas de un contenido impactante que hace falta digerir poco a poco analizando con ellas cómo se han sentido después del desarrollo de la actividad. Se puede decir que hay cuórum entre las mujeres al expresar que ha sido de su agrado llevarlas a cabo, porque sienten el espacio como suyo, en plena compañía de personas con las que hacer el camino y expresar las emociones sin miedos.

Es importante tomar conciencia de lo que significa la ayuda social a estas personas. EL apoyo de muchos sectores para que puedan salir adelante, es fundamental que la cadena no se rompa y que tengan apoyo, a nivel asistencial, jurídico, psicológico, emocional, terapéutico, para poder restablecer sus vidas siendo un hecho real. No vale tan solo con trabajos bien hechos en ciertos campos, sino que toda la ayuda en su proceso se haga en condiciones para que tengan una integración social plena y no se desmorone, todos aquellos trabajos que iniciaron y fueron costosos. Las terapias desde los distintos campos del arte, refuerzan notablemente sus ganas de vivir y sus ilusiones, les dan estrategias para enfrentarse a las situaciones, y herramientas para que se inserten en la sociedad. En definitiva es una manera de empoderar a la mujer para que su vida nue dignidad y sentido.

\section{Conclusiones}

La combinación de diferentes formas de arte dinamiza el desarrollo de propuestas artísticas que ayudan a crear espacios de felicidad y alegría. Se generan climas de confianza, de respeto y de responsabilidad entre las mujeres, que proporcionan aprendizajes útiles para salir adelante en el día a día y en su relación con el mundo. La sensación de terminar un programa de estas características es muy positiva, ya que las mujeres y el equipo que trabaja con ellas coinciden en los avances que se obtienen de un trabajo bien hecho, donde se mejora en primer lugar la autoestima, la nueva forma de presentarse al mundo y el refuerzo en una comunicación asertiva con los demás. Todo ello son ingredientes que valen para comenzar una nueva forma de plantearse la vida y un afrontamiento de aquellos retos que se propongan.

\section{Referencias bibliográficas}

Alonso Grijalva, E. (2007). Mujeres víctimas de violencia doméstica con trastorno de estrés postraumático: validación empírica de un programa de tratamiento. Tesis Doctoral Universidad Complutense de Madrid.

Callejón, M. \& Granados, I. (2003). Creatividad, Expresión Y Arte: Terapia Para Una Educación Del Siglo XXI. Un Recurso Para La Integración Escuela Abierta: Revista De Investigación Educativa, 6, 129-147.

Córdoba, M. V. (2013). Violencia Sexual y empatía: la danza en contextos terapéuticos. Pensamiento Psicológico, 11(2), 177-190.

Damasio, A. R. (2001). La Sensación de lo que ocurre-cuerpo y emoción en la construcción de la consciencia. Editorial Debate: Madrid. 
Descartes, R. (2006). Las pasiones del alma, Madrid: Tecnos.

García, S. (2012). De regreso al cuerpo: propuesta psicoeducativa con bioenergética y danza movimiento terapia para mejorar la salud de mujeres víctimas de violencia sexual. Tesina del máster en danza movimiento terapia. Universidad Autónoma de Barcelona. Barcelona, España.

Garrido, S., Baker, F. A., Davidson, J. W., Moore, G., \& Wasserman, S. (2015). Music and trauma: The relationship between music, personality, and coping style. Frontiers in Psychology, 6, Article ID 977.

Goldie, P. (2002). The Emotions. A Philosophical Exploration, Oxford: Clarendon Press.

James, W. (1991). Principles of psychology. University of Chicago Press: Chicago.

Lynn, A. (2001). The Body remembers: dance movement therapy with an adult survivor of torture. American Journal of Dance Therapy, 23(1), 29-43.

Ojeda. M; Serrano, A. (2008). Papeles de arteterapia y educación artística para la inclusión social 157 Vol. 3/ 2008 (págs: 157-164).

Otero Caicedo, L.; Fernández Moreno, A. (2017). Ser en el arte: caminos de reconocimiento, en Arteterapia. Papeles de arteterapia y educación para inclusión social 12, 25-42.

Panhofer, H. (2005). Cuando las palabras no son suficientes. Fundamentos de la Danza Movimiento Terapia (DMT). En Terapias creativas. Universidad de Salamanca.

Prinz, J. (2004). Gut Reactions. A perceptual Theory of Emotion. New York: Oxford University Press.

Taylor, G. (1985). Pride, Shame and Guilt. Emotions of Self-assessment. Oxford: Clarendon Press. 